\title{
Traveling waves for models of phase transitions of solids driven by configurational forces
}

\author{
Shuichi Kawashima ${ }^{1 *}$ And Peicheng $\mathrm{ZHU}^{2,3 \dagger}$ \\ ${ }^{1}$ Graduate School of Mathematics, Kyushu University \\ Fukuoka 812-8581, Japan \\ and \\ ${ }^{2}$ Basque Center for Applied Mathematics \\ Building 500, Bizkaia Technology Park \\ E-48160 Derio, Spain \\ ${ }^{3}$ IKERBASQUE, Basque Foundation for Science \\ E-48011 Bilbao, Spain
}

\begin{abstract}
This article is concerned with the existence of traveling wave solutions, including standing waves, to some models based on configurational forces, describing respectively the diffusionless phase transformations of solid materials, e.g., Steel, and phase transitions due to interface motion by interface diffusion, e.g., Sintering. These models were proposed by Alber and Zhu in [3. We consider both the order-parameter-conserved case and the non-conserved one, under suitable assumptions. Also we compare our results with the corresponding ones for the Allen-Cahn and the Cahn-Hilliard equations coupled with linear elasticity, which are models for diffusion-dominated phase transformations in elastic solids.
\end{abstract}

\section{Introduction}

In his work [2], H.-D. Alber started the study of models of sharp interface describing phase transitions in alloys. Such models are of Hamiltonian type and complex from both theoretical and numerical viewpoints. Thus in [3, 4], as an approximation of those models, the phase field models were derived from the corresponding sharp interface ones for describing phase transitions in elastically deformable solids. The material under consideration possesses microstructures, and its phases are characterized by the structure of the crystal lattice, in which the atoms are arranged. An interface between different material phases moves if the crystal lattice is transformed from one structure to the other across the interface.

Often phase transformations are triggered by diffusion processes. Two well known models for diffusion dominated transformations are the Allen-Cahn and Cahn-Hilliard equations (see [7, 11, 12]).

\footnotetext{
*E-mail: kawashim@math.kyushu-u.ac.jp

${ }^{\dagger}$ E-mail: zhu@bcamath.org
} 
In this article we shall consider two models, one describing diffusionless transformations (also called martensitic transformations, cf. [18, p. 162]) of solid materials, e.g., Steel, and another for phase transitions due to interface motion by interface diffusion, e.g., Sintering. The derivation of these two models can be found in [3, 4]. Both models are based on a model for phase transitions driven by configurational forces, see [1]. For more details on configurational forces, we refer the reader to the book by Gurtin [16], and the references cited therein. The models studied here are also related to the work by Mullins [20] on the development of grooves. Some mathematical results have been obtained for special cases of those two types of models, see [3, 4, 5] for the existence of weak solutions, and [6] for the justification of the diffusive models by using asymptotic expansion methods.

In the present paper, we study the existence of traveling wave solutions, including standing wave solutions, for the phase field models in [3, 4]. The models are formulated for materials with two different phases, say, phase 1 and phase 2 , in which the order parameter $v=v(x, t) \in \mathbb{R}$ is assumed to take values near $v_{-}$or $v_{+}$if the material is in the phase 1 or 2 , respectively, where we assume, without loss of generality, that $v_{-}<v_{+}$, and $x=\left(x_{1}, \cdots, x_{n}\right) \in \mathbb{R}^{n}$ denotes the material point and $t \geq 0$ is the time variable. Let $u=\left(u^{i}(x, t)\right) \in \mathbb{R}^{n}$ be the displacement of the material. Then the first model in which the order parameter is not conserved is formulated as

$$
\begin{gathered}
-\operatorname{div}_{x} T=b \\
v_{t}=-\left(\psi_{v}(\varepsilon, v)-\Delta_{x} v\right)\left|\nabla_{x} v\right| .
\end{gathered}
$$

The second model is as follows

$$
\begin{gathered}
-\operatorname{div}_{x} T=b, \\
v_{t}=\operatorname{div}_{x}\left\{\nabla_{x}\left(\psi_{v}(\varepsilon, v)-\Delta_{x} v\right)\left|\nabla_{x} v\right|\right\} .
\end{gathered}
$$

In the latter, the order parameter is conserved. Eq. (1.4) can be written in the form of a conservation law:

$$
v_{t}=\operatorname{div}_{x} \mathbf{J},
$$

where $\mathbf{J}$ is a flux defined by

$$
\mathbf{J}=\nabla_{x}\left(\psi_{v}(\varepsilon, v)-\Delta_{x} v\right)\left|\nabla_{x} v\right|
$$

However 1.2 can not be rewritten in the conservative form.

We give now a more detailed description of the above systems. The symbols $\operatorname{div}_{x}, \nabla_{x}$ and $\Delta_{x}$ denote the divergence, the gradient and the Laplacian with respect to $x \in \mathbb{R}^{n}$. The $\varepsilon=\left(\varepsilon_{i j}\right) \in \mathcal{S}^{n}$ denotes the strain tensor which is given by

$$
\varepsilon_{i j}=\frac{1}{2}\left(u_{x_{j}}^{i}+u_{x_{i}}^{j}\right)
$$

where $\mathcal{S}^{n}$ denotes the set of all $n \times n$ real symmetric matrices. $T=\left(T_{i j}\right) \in \mathcal{S}^{n}$ is the Cauchy stress tensor and is given explicitly in terms of $(\varepsilon, v)$, see (1.6) below. Also, $\psi(\varepsilon, v)$ is a part of the free energy $\psi^{*}=\psi(\varepsilon, v)+\frac{1}{2}\left|\nabla_{x} v\right|^{2}$, which is given in (1.7) below, and $\psi_{v}(\varepsilon, v)$ denotes the derivative of $\psi(\varepsilon, v)$ with respect to $v$. Finally, $b=b(x, t)$ denotes the volume force which is assumed to be a given function of $(x, t)$. 
To close the systems, we need to add the following constitutive relations:

(CR1)

$$
T=D(\varepsilon-\bar{\varepsilon}(v)),
$$

(CR2)

$$
\psi(\varepsilon, v)=\frac{1}{2} D(\varepsilon-\bar{\varepsilon}(v)):(\varepsilon-\bar{\varepsilon}(v))+f(v) .
$$

Here $D=\left(D_{k l}^{i j}\right)$ is the elasticity tensor which is assumed to be a linear symmetric positive definite operator from $\mathcal{S}^{n}$ into $\mathcal{S}^{n}$; this means that for any $\sigma=\left(\sigma_{i j}\right) \in \mathcal{S}^{n}$,

$$
D \sigma=\left(\sum_{k, l=1}^{n} D_{k l}^{i j} \sigma_{k l}\right) \in \mathcal{S}^{n}, \quad(D \sigma): \sigma \geq c|\sigma|^{2}
$$

for some positive constant $c$. Here we denote the scalar product of two matrices $\sigma$ and $\tau$ by $\sigma: \tau=\sum_{i, j=1}^{n} \sigma_{i j} \tau_{i j}$, and the corresponding norm by $|\sigma|=(\sigma: \sigma)^{1 / 2}$. Also, $\bar{\varepsilon}(v) \in \mathcal{S}^{n}$ is assumed to be linear in $v$ :

$$
\bar{\varepsilon}(v)=\varepsilon^{(0)}+\varepsilon^{(1)} v,
$$

where $\varepsilon^{(0)}=\left(\varepsilon_{i j}^{(0)}\right) \in \mathcal{S}^{n}$ and $\varepsilon^{(1)}=\left(\varepsilon_{i j}^{(1)}\right) \in \mathcal{S}^{n}$ are transformation tensors which are assumed to be constant matrices, and $f(v)$ is a given smooth double-well potential with two local minima at $v=v_{-}$and $v=v_{+}$. Consequently, the pair $(v, u)(x, t)$ or $(v, \varepsilon)(x, t)$ is the unknown of our systems $(1.1)-(1.2)$ and $(1.3)-(1.4)$.

We can slightly rewrite our systems. A simple computation, using (1.6) and (1.7), yields

$$
\psi_{v}(\varepsilon, v)=-D(\varepsilon-\bar{\varepsilon}(v)): \varepsilon^{(1)}+f^{\prime}(v)=-T: \varepsilon^{(1)}+f^{\prime}(v),
$$

where $f^{\prime}$ denotes the derivative of $f$. Therefore the systems become

$$
\begin{gathered}
-\operatorname{div}_{x} T=b, \\
v_{t}=\left(T: \varepsilon^{(1)}-f^{\prime}(v)+\Delta_{x} v\right)\left|\nabla_{x} v\right|,
\end{gathered}
$$

and

$$
\begin{gathered}
-\operatorname{div}_{x} T=b, \\
v_{t}=-\operatorname{div}_{x}\left\{\nabla_{x}\left(T: \varepsilon^{(1)}-f^{\prime}(v)+\Delta_{x} v\right)\left|\nabla_{x} v\right|\right\},
\end{gathered}
$$

respectively. The evolution equations (1.10) and (1.12) for the order parameter $v$ are nonuniformly parabolic because of the degenerate terms $\left|\nabla_{x} v\right| \Delta_{x} v$ and $\operatorname{div}_{x}\left(\nabla_{x} \Delta_{x} v\left|\nabla_{x} v\right|\right)$, respectively.

The main purpose of this article is to study the existence of traveling wave and standing wave solutions to the systems $(1.9)-(1.10)$ and $(1.11)-(1.12)$. To this end, we assume that the volume force $b=b(x, t)$ is identically zero, i.e., $b \equiv 0$, and look for one-dimensional solutions of the form

$$
v=v\left(x_{1}, t\right), \quad u=\left(u^{1}\left(x_{1}, t\right), 0, \cdots, 0\right) .
$$


Under suitable structural assumptions, it will be observed that our $n$-dimensional systems are essentially reduced to the one-dimensional equations

$$
v_{t}=\left(\mu-f^{\prime}(v)+v_{x_{1} x_{1}}\right)\left|v_{x_{1}}\right|
$$

and

$$
v_{t}=-\left\{\left(-f^{\prime}(v)+v_{x_{1} x_{1}}\right)_{x_{1}}\left|v_{x_{1}}\right|\right\}_{x_{1}}
$$

respectively, where $\mu$ is a constant to be determined. Moreover, we will show that these one-dimensional equations (1.13) and (1.14 have a traveling wave solution $v=v\left(x_{1}-s t\right)$ and a standing wave solution $v=v\left(x_{1}\right)$, respectively, both connecting the phase states $v_{-}$and $v_{+}$, provided that $f\left(v_{+}\right)=f\left(v_{-}\right)$. A comparison in Section 4, will show that this existence result of standing wave solutions to (1.14) is the same to the known results for the Cahn-Hilliard equation, however if $\mu \neq 0$, results of traveling waves to (1.13) are quite different from those for Allen-Cahn equation.

The remaining parts of this article are as follows: In Section 2, under suitable structural assumptions, we shall reduce the multi-dimensional systems into the onedimensional ones, which can be finally reduced to the one single equation (1.13) or (1.14) for each case. Then we shall investigate, in Section 3, the existence of traveling wave and standing wave solutions for these reduced one-dimensional systems. The last section is devoted to the interesting comparison of our results with the existing ones for the Allen-Cahn or Cahn-Hilliard equation coupled with linear elasticity.

\section{Reduction to one-dimensional equations}

In this section, under suitable structural assumptions, we reduce our multi-dimensional systems $(1.9)-(1.10)$ and $(1.11)-(1.12)$ into the corresponding ones in the one-dimensional form. To this end, we first assume that the volume force $b=b(x, t)$ is identically zero, namely,

$$
b \equiv 0
$$

and look for one-dimensional solutions of the form

$$
v=v\left(x_{1}, t\right), \quad u=\left(u^{1}\left(x_{1}, t\right), 0, \cdots, 0\right) .
$$

We now calculate the Cauchy stress tensor $T=\left(T_{i j}\right)$ for these particular solutions. For $u=\left(u^{1}\left(x_{1}, t\right), 0, \cdots, 0\right)$, the strain tensor $\varepsilon=\left(\varepsilon_{i j}\right)$ has the simplest form

$$
\varepsilon_{11}=u_{x_{1}}^{1}, \quad \varepsilon_{i j}=0 \text { for }(i, j) \neq(1,1) .
$$

Consequently, we obtain from $(1.6)$ and $(1.8)$ that

$$
\begin{aligned}
T_{i j} & =D^{i j}:\left(\varepsilon-\left(\varepsilon^{(0)}+\varepsilon^{(1)} v\right)\right) \\
& =D_{11}^{i j} u_{x_{1}}^{1}-\left(D^{i j}: \varepsilon^{(0)}\right)-\left(D^{i j}: \varepsilon^{(1)}\right) v,
\end{aligned}
$$

where $D^{i j}=\left(D_{k l}^{i j}\right) \in \mathcal{S}^{n}$. In particular, we have

$$
T_{11}=D_{11}^{11} u_{x_{1}}^{1}-\left(D^{11}: \varepsilon^{(0)}\right)-\left(D^{11}: \varepsilon^{(1)}\right) v .
$$


Eliminating $u_{x_{1}}^{1}$ in (2.4) by using (2.5) yields an expression of $T$ in terms of $T_{11}$ and $v$. Namely, we obtain

$$
T=\sigma T_{11}+\tau^{(0)}+\tau^{(1)} v
$$

where $\sigma=\left(\sigma_{i j}\right), \tau^{(0)}=\left(\tau_{i j}^{(0)}\right)$ and $\tau^{(1)}=\left(\tau_{i j}^{(1)}\right)$ are the constant tensors in $\mathcal{S}^{n}$ given explicitly as

$$
\begin{aligned}
\sigma_{i j} & =D_{11}^{i j} / D_{11}^{11}, \\
\tau_{i j}^{(m)} & =\left(1 / D_{11}^{11}\right)\left\{D_{11}^{i j}\left(D^{11}: \varepsilon^{(m)}\right)-D_{11}^{11}\left(D^{i j}: \varepsilon^{(m)}\right)\right\}, \quad m=0,1 .
\end{aligned}
$$

In fact, we have from (2.4) and (2.5) that

$$
\begin{aligned}
T_{i j} & =\left(D_{11}^{i j} / D_{11}^{11}\right)\left\{T_{11}+\left(D^{11}: \varepsilon^{(0)}\right)+\left(D^{11}: \varepsilon^{(1)}\right) v\right\}-\left(D^{i j}: \varepsilon^{(0)}\right)-\left(D^{i j}: \varepsilon^{(1)}\right) v \\
& =\sigma_{i j} T_{11}+\tau_{i j}^{(0)}+\tau_{i j}^{(1)} v
\end{aligned}
$$

which gives the expression $(2.6)$. Notice that $\sigma_{11}=1$ and $\tau_{11}^{(0)}=\tau_{11}^{(1)}=0$. Also, using (2.6), we see that

$$
T: \varepsilon^{(1)}=\alpha T_{11}+\beta+\gamma v
$$

where $\alpha, \beta$ and $\gamma$ are the constants given by

$$
\begin{aligned}
& \alpha=\sigma: \varepsilon^{(1)}=\left(1 / D_{11}^{11}\right)\left(D_{11}: \varepsilon^{(1)}\right) \\
& \beta=\tau^{(0)}: \varepsilon^{(1)}=\left(1 / D_{11}^{11}\right)\left\{\left(D_{11}: \varepsilon^{(1)}\right)\left(D^{11}: \varepsilon^{(0)}\right)-D_{11}^{11}\left(D \varepsilon^{(0)}: \varepsilon^{(1)}\right)\right\} \\
& \gamma=\tau^{(1)}: \varepsilon^{(1)}=\left(1 / D_{11}^{11}\right)\left\{\left(D_{11}: \varepsilon^{(1)}\right)\left(D^{11}: \varepsilon^{(1)}\right)-D_{11}^{11}\left(D \varepsilon^{(1)}: \varepsilon^{(1)}\right)\right\}
\end{aligned}
$$

with $D_{11}=\left(D_{11}^{i j}\right) \in \mathcal{S}^{n}$. These observations are summarized as follows.

Lemma 2.1 Let $u=\left(u^{1}\left(x_{1}, t\right), 0, \cdots, 0\right)$. Then the Cauchy stress tensor $T=\left(T_{i j}\right)$ has the form 2.6), in which the component $T_{11}$ is given by 2.5. Moreover, we have (2.8).

Now, we assume the following structural conditions:

(A1)

$$
D_{11}^{11}\left(D^{i 1}: \varepsilon^{(1)}\right)=D_{11}^{i 1}\left(D^{11}: \varepsilon^{(1)}\right) \text { for } i=1, \cdots, n
$$

(A2)

$$
\left(D_{11}: \varepsilon^{(1)}\right)\left(D^{11}: \varepsilon^{(1)}\right)=D_{11}^{11}\left(D \varepsilon^{(1)}: \varepsilon^{(1)}\right)
$$

Notice that 2.10 implies $\tau_{i 1}^{(1)}=0$ for $i=1, \cdots, n$ in 2.7), while 2.11) gives $\gamma=0$ in 2.9. It then follows that

$$
\begin{gathered}
T_{i 1}=\sigma_{i 1} T_{11}+\tau_{i 1}^{(0)}, \quad i=1, \cdots, n, \\
T: \varepsilon^{(1)}=\alpha T_{11}+\beta .
\end{gathered}
$$


This implies that the first column of $T$ is constant if and only if $T_{11}$ in 2.5$)$ is constant. Moreover, in this case, $T: \varepsilon^{(1)}$ becomes a constant too. Therefore, under the assumptions (2.10) and (2.11), for one-dimensional solutions of the form $(2.2)$, we can reduce the original multi-dimensional systems $(1.9)-(1.10)$ and 1.11$)-(1.12)$ both with $b \equiv 0$ into the following one-dimensional systems:

$$
\begin{gathered}
T_{11}=D_{11}^{11} u_{x_{1}}^{1}-\left(D^{11}: \varepsilon^{(0)}\right)-\left(D^{11}: \varepsilon^{(1)}\right) v \\
v_{t}=\left(\alpha T_{11}+\beta-f^{\prime}(v)+v_{x_{1} x_{1}}\right)\left|v_{x_{1}}\right|
\end{gathered}
$$

and

$$
\begin{gathered}
T_{11}=D_{11}^{11} u_{x_{1}}^{1}-\left(D^{11}: \varepsilon^{(0)}\right)-\left(D^{11}: \varepsilon^{(1)}\right) v \\
v_{t}=-\left\{\left(-f^{\prime}(v)+v_{x_{1} x_{1}}\right)_{x_{1}}\left|v_{x_{1}}\right|\right\}_{x_{1}},
\end{gathered}
$$

respectively, where $T_{11}$ is a constant. These considerations are summarized as follows.

Proposition 2.2 Suppose that the assumptions (2.10) and (2.11) are met. Then the function $(v, u)$ given by $(2.2)$ is a solution to system (1.9) - 1.10) (resp. system (1.11) - (1.12) with $b \equiv 0$ if and only if $\left(v, u^{1}\right)$ satisfies the one-dimensional system (2.14) 2.15) (resp. the system 2.16 - 2.17) with $T_{11}$ being a constant.

Remark 1. Since $T_{11}$ is regarded as a given constant parameter, both systems (2.14) (2.15) and (2.16) - (2.17) are completely decoupled. This means that we solve 2.15$)$ or 2.17) for $v$ independently, and then find $u^{1}$ from (2.14) or 2.16).

\section{Traveling wave solutions}

In this section we are going to look for smooth traveling wave solutions to the onedimensional systems $2.14-2.15$ and 2.16 - 2.17) in the form

$$
v=v\left(x_{1}-s t\right), \quad u_{x_{1}}^{1}=w\left(x_{1}-s t\right),
$$

where $s$ is a constant velocity to be determined. Here it is assumed that $v(\xi)$ and $w(\xi)$ connect the constant states $v_{ \pm}\left(v_{-}<v_{+}\right)$and $w_{ \pm}$(to be determined), respectively:

$$
v(\xi) \rightarrow v_{ \pm}, \quad w(\xi) \rightarrow w_{ \pm}
$$

as $\xi \rightarrow \pm \infty$, and satisfies the requirement

$$
v_{\xi}>0
$$

for $\xi \in \mathbb{R}$. Here we have written $\xi=x_{1}-s t$.

For the potential $f(v)$, we impose the following conditions:

Assumption (B)

$f(v)$ is a smooth double-well potential which has two local minima at $v_{-}$and $v_{+}$with $v_{-}<v_{+}$and one local maximum at $v_{*}$ with $v_{-}<v_{*}<v_{+}$, and satisfies $f^{\prime}(v)>0$ for $v_{-}<v<v_{*}$ and $f^{\prime}(v)<0$ for $v_{*}<v<v_{+}$. 
For simplicity, we assume that

$$
\begin{aligned}
& f^{(k)}\left(v_{+}\right)=0 \text { for } 1 \leq k \leq 2 m_{1}-1, \quad f^{\left(2 m_{1}\right)}\left(v_{+}\right)>0, \\
& f^{(k)}\left(v_{-}\right)=0 \text { for } 1 \leq k \leq 2 m_{2}-1, \quad f^{\left(2 m_{2}\right)}\left(v_{-}\right)>0
\end{aligned}
$$

where $m_{1}$ and $m_{2}$ are positive integers. A straightforward computation shows that for solutions of the form (3.1), system (2.14) - 2.15$)$ turns out to be

$$
\begin{gathered}
T_{11}=D_{11}^{11} w-\left(D^{11}: \varepsilon^{(0)}\right)-\left(D^{11}: \varepsilon^{(1)}\right) v, \\
-s v_{\xi}=\left(\alpha T_{11}+\beta-f^{\prime}(v)+v_{\xi \xi}\right)\left|v_{\xi}\right|
\end{gathered}
$$

while system $2.16-2.17$ becomes

$$
\begin{gathered}
T_{11}=D_{11}^{11} w-\left(D^{11}: \varepsilon^{(0)}\right)-\left(D^{11}: \varepsilon^{(1)}\right) v, \\
-s v_{\xi}=-\left\{\left(-f^{\prime}(v)+v_{\xi \xi}\right)_{\xi}\left|v_{\xi}\right|\right\}_{\xi},
\end{gathered}
$$

where $T_{11}$ is assumed to be a given constant.

Remark 2. Since systems 2.14 -2.15 and $(2.16)-(2.17)$ are intended to describe phase transitions, it is necessary to assume that the nonlinearity $f(v)$ has at least two different minima, say $v_{-}, v_{+}$and one maximum, say $v_{*}$.

We now consider the case that $v_{-}<v_{+}$(The case that $v_{-}>v_{+}$can be treated in a similar manner, and we conclude that traveling wave exists if and only if $v_{\xi}<0$ is satisfied). In this case, traveling wave exists if and only if (3.3) is true. In this remark, we prove the necessity part. The sufficiency part is the main task of the present article and will be proved after this remark.

Assume that there exists a smooth traveling wave $v$ and

$$
\text { there exists at least one point, say } \xi_{0} \in \mathbb{R} \text {, such that } v^{\prime}\left(\xi_{0}\right)>0 \text {. }
$$

Otherwise, $v^{\prime}(\xi) \leq 0$ for all $\xi \in \mathbb{R}$. It is easy to see that such $v$ can not connect, $v_{-}$and $v_{+}$, at minus and plus infinity, respectively, provided that $v_{-}<v_{+}$.

Define

$$
\begin{aligned}
\mathcal{N}_{\xi_{0}}=\left\{N\left(\xi_{0}\right) \subset \mathbb{R} \mid\right. & N\left(\xi_{0}\right) \text { any open simply connected set containing } \xi_{0}, v^{\prime}(\xi) \geq 0, \\
& \left.\forall \xi \in N\left(\xi_{0}\right), \text { and } v^{\prime}(\xi)=0 \text { only if } \xi \text { is an inflection point }\right\}(3.11)
\end{aligned}
$$

and a relation $\prec$ : for any two elements $N_{1}, N_{2} \in \mathcal{N}_{\xi_{0}}, N_{1} \prec N_{2}$ means $N_{1} \subset N_{2}$. By Zorn's lemma (see, e.g. [27]) we conclude that there exists a maximal element $N_{\max }\left(\xi_{0}\right)$ in $\mathcal{N}_{\xi_{0}}$, and assume that there exist two numbers $\xi_{-}, \xi_{+}$such that $N_{\max }\left(\xi_{0}\right)=\left(\xi_{-}, \xi_{+}\right)$. It is easy to see that $v^{\prime}\left(\xi_{ \pm}\right)=0$, and we assume that

$$
v\left(\xi_{-}\right)=\underline{v}, \quad v\left(\xi_{+}\right)=\bar{v}
$$

with $\underline{v}, \bar{v} \in \mathbb{R}$ and $\underline{v}<\bar{v}$. In $\left(\xi_{-}, \xi_{+}\right)$, equation (3.7) can be reduced to an ordinary differential equation of second order as follows

$$
v_{\xi \xi}=f^{\prime}(v)-\left(s+\alpha T_{11}+\beta\right) .
$$


I) Suppose that both $\xi_{+}$and $\xi_{-}$are infinite. Thus we can obtain $\bar{v}=v_{+}$and $\underline{v}=v_{-}$. Invoking assumption (3.4), we rewrite (3.7) as

$$
v_{\xi}=b(v)\left(v_{+}-v\right)^{m_{1}}\left(v-v_{-}\right)^{m_{2}}, \text { for } \xi \in\left(\xi_{-}, \xi_{+}\right)
$$

here $b(v)$ is a smooth function. Thus from uniqueness theorem of ordinary differential equations (see e.g. page 259, 24]), we exclude the case that $v_{\xi}\left(\xi_{0}\right)=0$ for some finite $\xi_{0} \in \mathbb{R}$, and assumption 3.3 follows.

II) Assume $\bar{v}>v_{+}\left(\right.$or $\left.\underline{v}<v_{-}\right)$at a finite point. Then there exists a point, say $\xi_{*}$, such that $v\left(\xi_{*}\right)=v_{+}$. As in I) we know that $v$ satisfies (3.14) in a small interval $\left[\xi_{*}, \xi^{*}\right)$ with $\xi^{*}>\xi_{*}$. This is impossible again by uniqueness theorem of ordinary differential equations.

III) Now we assume that either $\xi_{+}$or $\xi_{-}$is finite (We only consider the case $\xi_{+}$is finite, another case can be treated in a similar way). Then there is a suitable number $\xi_{*}>\xi_{+}$such that

i) $v_{\xi}(\xi)<0$ for $\xi \in\left(\xi_{+}, \xi_{*}\right)$;

ii) $v(\xi)=\bar{v}$ for $\xi \in\left(\xi_{+}, \xi_{*}\right)$,

as shown in Figure 1 (see page 16 of this article).

We first handle case ii). Since we look for a smooth solution $v$, the left limits at $\xi_{+}$ of derivatives up to second order (which is enough here) of $v$ must be equal to the right ones, respectively. It is easy to compute that $v_{\xi \xi}\left(\xi_{+}+0\right)=0$. Thus taking limit for both sides of 3.13 yields that

$$
0=v_{\xi \xi}\left(\xi_{-}-0\right)=f^{\prime}(\bar{v})-\left(s+\alpha T_{11}+\beta\right) .
$$

So this means $v \equiv \bar{v}$ is a solution to (3.13). This violates uniqueness theorem of ordinary differential equations in $\left(\xi_{-}, \xi_{+}\right]$. Therefore case ii) could not happen.

It remains to consider case i). In $\left(\xi_{+}, \xi^{*}\right)$, equation (3.7) is equivalent to

$$
v_{\xi \xi}=f^{\prime}(v)-\left(-s+\alpha T_{11}+\beta\right) .
$$

Thus from (3.16) and 3.13 one has

$$
f^{\prime}(\bar{v})-\left(-s+\alpha T_{11}+\beta\right)=f^{\prime}(\bar{v})-\left(s+\alpha T_{11}+\beta\right)
$$

which implies that

$$
s=0,
$$

hence if $s \neq 0$, then case i) can not be true. Suppose that (3.17) is satisfied, then (3.16) and (3.13) become

$$
\begin{aligned}
& \frac{1}{2}\left(v_{\xi}\right)^{2}=\Phi(v ; c):=f(v)-\left(\alpha T_{11}+\beta\right) v+c, \text { for } \xi \in\left(\xi_{-}, \xi_{+}\right) \\
& \frac{1}{2}\left(v_{\xi}\right)^{2}=\Phi\left(v ; c_{1}\right):=f(v)-\left(\alpha T_{11}+\beta\right) v+c_{1}, \text { for } \xi \in\left(\xi_{+}, \xi^{*}\right) .
\end{aligned}
$$

We can not see immediately that equations (3.18) and (3.19) can be rewritten in the form of (3.14) that meets the conditions of uniqueness theorem of first order ordinary differential equations. Note that the right hand side of (3.18) differs, by a constant, from 
that of (3.19), namely $c, c_{1}$ may be different. But at $\xi=\xi_{+}$, there hold $v_{\xi}=0, v=\bar{v}$. Hence, it follows from (3.18) and (3.19) that $\Phi(\bar{v} ; c)=0=\Phi\left(\bar{v} ; c_{1}\right)$, so $c=c_{1}$. That is to say $v$ satisfies the same equation in both $\left(\xi_{+}, \xi^{*}\right)$ and $\left(\xi_{-}, \xi_{+}\right)$. By extension, it is also true that $v$ satisfies the same equation in any interval contained in $(-\infty, \infty)$. Thus $c=c_{1}=-\left(f\left(v_{ \pm}\right)-\left(s+\alpha T_{11}+\beta\right) v_{ \pm}\right)$, and (3.7) can be rewritten as (3.14). We can apply again uniqueness theorem of ordinary differential equations and conclude it is impossible that $v_{\xi}\left(\xi_{0}\right)=0$ for any finite $\xi_{0}$. This leads to a contradiction since we assumed that $v_{\xi}\left(\xi_{+}\right)=0$ and $\xi_{+}$is finite. So we again obtain that $v_{\xi}$ can not change its sign even (3.17) holds, and must be positive since we assume $v_{-}<v_{+}$.

We now turn back to the investigation of traveling waves. The remaining of this section will be divided into two parts corresponding to systems $(3.6)-(3.7)$ and $(3.8)-$ (3.9), respectively.

Part 1. We first study the non-conservative system (3.6) - (3.7). Letting $\xi \rightarrow \pm \infty$ in (3.6) and recalling that $T_{11}$ is constant, we obtain

$$
T_{11}=D_{11}^{11} w_{ \pm}-\left(D^{11}: \varepsilon^{(0)}\right)-\left(D^{11}: \varepsilon^{(1)}\right) v_{ \pm}
$$

which gives

$$
D_{11}^{11}\left(w_{+}-w_{-}\right)=\left(D^{11}: \varepsilon^{(1)}\right)\left(v_{+}-v_{-}\right) .
$$

On the other hand, since we have assumed (3.3), one can obtain from (3.7) that

$$
\begin{aligned}
-s v_{\xi} & =\left(\alpha T_{11}+\beta-f^{\prime}(v)+v_{\xi \xi}\right) v_{\xi} \\
& =\left\{\left(\alpha T_{11}+\beta\right) v-f(v)+\frac{1}{2}\left(v_{\xi}\right)^{2}\right\}_{\xi} .
\end{aligned}
$$

Integrating it with respect to $\xi$ yields

$$
\frac{1}{2}\left(v_{\xi}\right)^{2}=f(v)-\left(s+\alpha T_{11}+\beta\right) v-A=: g(v),
$$

where $A$ is a constant. Letting $\xi \rightarrow \pm \infty$ and using (3.2) gives

$$
f\left(v_{ \pm}\right)-\left(s+\alpha T_{11}+\beta\right) v_{ \pm}-A=0,
$$

from which follows that

$$
s+\alpha T_{11}+\beta=\frac{f\left(v_{+}\right)-f\left(v_{-}\right)}{v_{+}-v_{-}} .
$$

As a consequence, we see that $g(v)$ defined in (3.21) can be rewritten as

$$
g(v)=f(v)-f\left(v_{ \pm}\right)-\frac{f\left(v_{+}\right)-f\left(v_{-}\right)}{v_{+}-v_{-}}\left(v-v_{ \pm}\right) .
$$

Since $g(v) \geq 0$ by (3.21), we get

$$
f(v)-f\left(v_{ \pm}\right) \geq \frac{f\left(v_{+}\right)-f\left(v_{-}\right)}{v_{+}-v_{-}}\left(v-v_{ \pm}\right)
$$


for all $v$ such that $v_{-}<v<v_{+}$. We divide this inequality by $v-v_{ \pm}$, where $v-v_{+}<0<$ $v-v_{-}$, and take the limits as $\xi \rightarrow \pm \infty$. Since $v(\xi) \rightarrow v_{ \pm}$as $\xi \rightarrow \pm \infty$, we deduce that

$$
f^{\prime}\left(v_{+}\right) \leq \frac{f\left(v_{+}\right)-f\left(v_{-}\right)}{v_{+}-v_{-}} \leq f^{\prime}\left(v_{-}\right) .
$$

By the assumption (3.4) or (3.5) we have $f^{\prime}\left(v_{ \pm}\right)=0$, and thus we infer that

$$
f\left(v_{+}\right)=f\left(v_{-}\right)
$$

which together with 3.22 gives

$$
s=-\left(\alpha T_{11}+\beta\right) .
$$

Thus the velocity $s$ of the traveling wave solution is determined.

Consequently, our equation 3.21 can be simplified to

$$
\frac{1}{2}\left(v_{\xi}\right)^{2}=f(v)-f\left(v_{ \pm}\right) .
$$

By (3.24) and the assumptions (3.4) and (3.5), we can write

$$
f(v)-f\left(v_{ \pm}\right)=\frac{1}{2} a(v)\left(v_{+}-v\right)^{2 m_{1}}\left(v-v_{-}\right)^{2 m_{2}}
$$

with $a(v)>0$ being a smooth function. Therefore $(3.26)$ can be rewritten as

$$
v_{\xi}=a(v)^{1 / 2}\left(v_{+}-v\right)^{m_{1}}\left(v-v_{-}\right)^{m_{2}} .
$$

This equation admits a smooth solution $v(\xi)$ satisfying $(3.2)$ and (3.3). Moreover, the solution verifies the following decay estimates for $\xi \rightarrow \pm \infty$ :

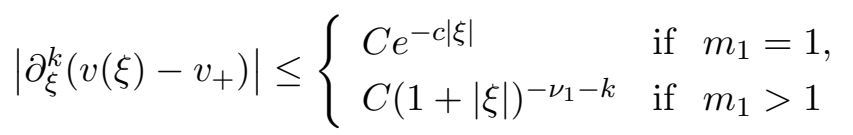

for $\xi \rightarrow \infty$, and

$$
\left|\partial_{\xi}^{k}\left(v(\xi)-v_{-}\right)\right| \leq \begin{cases}C e^{-c|\xi|} & \text { if } m_{2}=1 \\ C(1+|\xi|)^{-\nu_{2}-k} & \text { if } m_{2}>1\end{cases}
$$

for $\xi \rightarrow-\infty$, where $k$ is a non-negative integer, $\nu_{1}=1 /\left(m_{1}-1\right), \nu_{2}=1 /\left(m_{2}-1\right)$, and $C$ and $c$ are positive constants.

Thus we have proved the following theorem:

Theorem 3.1 (Non-conservative case) Suppose that the assumptions (3.4) and (3.5) are satisfied. Then the one-dimensional system (2.14) - 2.15) admits a smooth traveling wave solution $\left(v, u_{x_{1}}^{1}\right)=(v, w)\left(x_{1}-s t\right)$ satisfying $(3.2)$ and $(3.3)$ if and only if the constants $s, v_{ \pm}$and $w_{ \pm}$satisfy the relations (3.25), (3.24) and (3.20). In particular, two local minima $f\left(v_{ \pm}\right)$must coincide in this case. Moreover, such a traveling wave solution is unique up to a translation in $\xi$ and verifies the decay estimates 3.29) and 3.30 for $\xi \rightarrow \pm \infty$, respectively. 
Part 2. Next, we discuss the conservative system 3.8$)-(3.9)$. In this case, we still have (3.20). Also, integrating (3.9) gives

$$
-s v=-\left(-f^{\prime}(v)+v_{\xi \xi}\right)_{\xi}\left|v_{\xi}\right|+A_{1},
$$

where $A_{1}$ is a constant. Letting $\xi \rightarrow \pm \infty$, we then obtain $A_{1}=-s v_{ \pm}$, which implies

$$
s=0 .
$$

Whence we have $A_{1}=0$ so that (3.31) becomes $\left(-f^{\prime}(v)+v_{\xi \xi}\right)\left|v_{\xi}\right|=0$. Since $v_{\xi}>0$ by 3.3 , we get $\left(-f^{\prime}(v)+v_{\xi \xi}\right)_{\xi}=0$ and hence

$$
v_{\xi \xi}-f^{\prime}(v)=A_{2},
$$

where $A_{2}$ is a constant. Letting again $\xi \rightarrow \pm \infty$, we find that $A_{2}=-f^{\prime}\left(v_{ \pm}\right)=0$, where we have used the assumption (3.4) or (3.5). Consequently, (3.33) becomes

$$
v_{\xi \xi}-f^{\prime}(v)=0 .
$$

Multiplying (3.34) by $v_{\xi}$, we get $\left\{\frac{1}{2}\left(v_{\xi}\right)^{2}-f(v)\right\}_{\xi}=0$. We integrate this equation in $\xi$ to obtain

$$
\frac{1}{2}\left(v_{\xi}\right)^{2}=f(v)-A_{3}
$$

where $A_{3}$ is a constant. Letting $\xi \rightarrow \pm \infty$, we see that $A_{3}=f\left(v_{ \pm}\right)$and hence the relation (3.24) must be satisfied. Consequently, we find that (3.35) is just the same as (3.26) in Part 1. Therefore the same arguments as in Part 1 prove the following theorem.

Theorem 3.2 (Conservative case) Suppose that the assumptions (3.4) and (3.5) are satisfied. Then the one-dimensional system (2.16) - 2.17) admits a smooth traveling wave solution $\left(v, u_{x_{1}}^{1}\right)=(v, w)\left(x_{1}-s t\right)$ satisfying $(3.2)$ and (3.3) if and only if the constants $s$, $v_{ \pm}$and $w_{ \pm}$satisfy the relations (3.32), (3.24) and (3.20). In particular, two local minima $f\left(v_{ \pm}\right)$must coincide also in this case. In particular, necessarily, $s=0$, and the traveling wave is actually a standing wave solution. Moreover, such a standing wave solution is unique up to a translation in $\xi$ and verifies the decay estimates (3.29) and (3.30) for $\xi \rightarrow \pm \infty$, respectively.

\section{Comparison with the Allen-Cahn/Cahn-Hilliard equa- tions coupled with linear elasticity}

In this section we are going to compare our results with those for the Allen-Cahn and the Cahn-Hilliard equations, coupled with the linear elasticity system 1.9 with constitutive relation (1.6). We shall consider only quasi-static models. The Allen-Cahn and CahnHilliard equations coupled with $(1.9)$ are, respectively, as follows

$$
\begin{gathered}
-\operatorname{div}_{x} T=b, \\
v_{t}=T: \varepsilon^{(1)}-f^{\prime}(v)+\Delta_{x} v,
\end{gathered}
$$


and

$$
\begin{gathered}
-\operatorname{div}_{x} T=b, \\
v_{t}=-\operatorname{div}_{x}\left\{\nabla_{x}\left(T: \varepsilon^{(1)}-f^{\prime}(v)+\Delta_{x} v\right)\right\} .
\end{gathered}
$$

Here we made the same assumptions (1.6) and 1.7 for the systems.

As we will see, there are interesting differences between the result of existence of traveling waves for system $(4.1)-(4.2)$ and those for system $(1.1)-(1.2)$.

The well-posedness of systems (4.1) - (4.2) and (4.3) - (4.4), has been studied, in particular, in [10, 17].

Their is also an extensive literature on traveling waves to the single Allen-Cahn equation (4.2) with various nonlinearities: we refer the reader to the articles, e.g., Aronson and Weinberger [8], Berestycki and Hamel [9], [15], Ninomiya and Taniguchi [21, 22], Taniguchi [25], or the book by Fife [14], etc. However, there are very few results, to the knowledge of the authors, for the existence of traveling waves for the complete systems 4.1 - 4.2 and 4.3 - 4.4.

\subsection{Traveling waves for the Allen-Cahn/Cahn-Hilliard models}

We divide this subsection into two parts.

Part 1. System (4.1) - 4.2). We now turn to consider the non-conservative case. We assume that

$$
b=0, \quad v=v\left(x_{1}, t\right), \quad u=\left(u^{1}\left(x_{1}, t\right), 0, \cdots, 0\right),
$$

and that the same assumptions 2.10 - 2.11) are true to system (4.1) - 4.2) as in Section 2. Then similar computations yield that (4.1) - 4.2) can be reduced to

$$
v_{t}=v_{x_{1} x_{1}}+\mu-f^{\prime}(v),
$$

where $\mu=\alpha T_{11}+\beta$ is a constant.

To prove the existence of traveling wave solutions to 4.5$)$, we recall one of the existence theorems (see for instance, [19, 15] and also the references cited therein) for the following single Allen-Cahn equation

$$
v_{t}=v_{x_{1} x_{1}}+g(v) \text {. }
$$

Notice that eq. 4.5 can be written in the form of (4.6) by defining

$$
g(v)=\mu-f^{\prime}(v) .
$$

For the convenience of the readers, we state the following theorem for equation 4.6.

Theorem 4.1 (Kanel [19], and Theorem 3.1 in [15]) Let $v_{-}<v_{*}<v_{+}$and let $g \in C^{1}(\mathbb{R})$ satisfy $g\left(v_{ \pm}\right)=0, g^{\prime}\left(v_{ \pm}\right)<0, g(u)<0$ for $v_{-}<v<v_{*}$, and $g(v)>0$ for $v_{*}<v<v_{+}$. Then there exists a unique (up to a translation) monotone traveling wave solution to (4.6), which connects the end states $v_{-}$and $v_{+}$.

In the following, when we speak of a "traveling wave over $[\alpha, \beta]$ with velocity $s_{\alpha, \beta}$ ", we shall mean a solution of equation (4.6) with the given $s_{\alpha, \beta}$, which is positive in $(\alpha, \beta)$ and vanishes at $\alpha$ and $\beta$. We now cite the following theorem 
Theorem 4.2 (Theorem 2.7 in [15]) Assume that $g \in C^{1}(\mathbb{R})$ with $g\left(v_{ \pm}\right)=0$, and let there exist a traveling wave over $\left[v_{-}, v_{*}\right]$ with velocity $s_{v_{-}, v_{*}}$, and one over $\left[v_{*}, v_{+}\right]$with velocity $s_{v_{*}, v_{+}}<s_{v_{-}, v_{*}}$. Then there exists a traveling wave over $\left[v_{-}, v_{+}\right]$with velocity $s_{v_{-}, v_{+}}$satisfying

$$
s_{v_{*}, v_{+}}<s_{v_{-}, v_{+}}<s_{v_{-}, v_{*}} \text {. }
$$

Remark 3. In the original version of this theorem, $v_{-}$and $v_{+}$are assigned special values which are $v_{-}=0$ and $v_{+}=1$.

Remark 4. It has been pointed out in [25], p. 320, that, based upon Theorem 4.2, one can find a function $g$ such that for such $g$, eq. (4.6) has a traveling wave over $\left[v_{-}, v_{*}\right]$ with velocity $s_{v_{-}, v_{*}}$, and one over $\left[v_{*}, v_{+}\right]$with velocity $s_{v_{*}, v_{+}} \geq s_{v_{-}, v_{*}}$. The condition $s_{v_{*}, v_{+}}<s_{v_{-}, v_{*}}$ required by Theorem 4.2 is violated, so there exists no traveling wave that connects two states $v_{ \pm}$, to eq. (4.6).

From Theorem 4.1 we know that to guarantee the existence of traveling waves, the following necessary condition must be satisfied

$$
g\left(v_{ \pm}\right)=0
$$

Invoking that $v_{ \pm}$are two local minima of $f(v)$ by assumption (3.4), we have $f^{\prime}\left(v_{ \pm}\right)=0$, and thus there must hold

$$
0=g\left(v_{ \pm}\right)=\mu-f^{\prime}\left(v_{ \pm}\right)=\mu
$$

hence,

$$
\mu=\alpha T_{11}+\beta=0,
$$

which means that there is no contribution from the elastic energy to the equation (4.5).

Suppose now that $\mu=0$ is satisfied. It is easy to see that (4.5) is reduced to 4.6 with $g(v)=-f^{\prime}(v)$. Equation 4.6 is just the single Allen-Cahn equation and many existence theorems are applicable to it. For example, under the assumption (3.4), it is easy to check that all the conditions in Theorem 4.1 are met for $g(v)=-f^{\prime}(v)$. Then applying Theorem 4.1, we conclude that there exists a traveling wave solution to 4.5 with $\mu=\alpha T_{11}+\beta=0$, and this traveling wave connects two states $v_{-}$and $v_{+}$. But there exist some nonlinear functions $f(v)$ for which there exists no traveling waves to the single Allen-Cahn equation (4.6) with $g(v)=-f^{\prime}(v)$, as pointed out in Remark 3 .

Part 2. System 4.3 - 4.4 . Next we consider the conservative model (4.3) - 4.4). Assuming that

$$
b=0, \quad v=v\left(x_{1}, t\right), \quad u=\left(u^{1}\left(x_{1}, t\right), 0, \cdots, 0\right),
$$

and that the same assumptions 2.10 - 2.11 are true, we can reduce system (4.3) (4.4) to

$$
v_{t}=-\left(v_{x_{1} x_{1}}-f^{\prime}(v)\right)_{x_{1} x_{1}} .
$$

Note that the gradient term in (3.31) for the modified Cahn-Hilliard model (1.3) (1.4) does not influence essentially the results after equation (3.34) in Section 3. Therefore, same arguments can be carried out for eq. (4.9) and thus we conclude easily that there is no traveling wave solution with non-zero speed for the Cahn-Hilliard model (4.3) $-(4.4)$ too. 


\subsection{Comparison}

From Theorems 3.2, 3.3 and the arguments in Subsection 4.1, we are now able to draw the following conclusions.

\section{Conclusions:}

A) Non-conservative case:

(1) Suppose that $\alpha T_{11}+\beta \neq 0$ is met, which means that the elastic energy contributes to the total free energy; Then the elastic effect prevents the formation of traveling waves to the Allen-Cahn model (4.1) - 4.2. In contrast, the gradient term overcomes the effect due to the elastic energy and so there still exists a traveling wave to the modified Allen-Cahn model (1.1) - 1.2).

(2) Assume that there holds $\alpha T_{11}+\beta=0$. In this case there is no elastic energy. Under suitable assumptions on the nonlinearity, we can prove that there exists a traveling wave solution with non-zero speed both for the Allen-Cahn model $(4.1)-(4.2)$ and also for the modified Allen-Cahn one 1.1 - 1.2.

However, in some other cases (e.g. for a function $g$ such that the condition $s_{\alpha, v_{+}}<$ $s_{v_{-}, \alpha}$ in Theorem 4.2 is not met, see Remark 3 ), there exists no traveling wave solutions for models 4.1 - 4.2 and (1.1) - 1.2.

B) Conservative case:

There is no essential difference from the viewpoint of the existence of traveling waves since both the Cahn-Hilliard model $(4.3)$ - 4.4) and the modified one (1.3) - 1.4 admit only a standing wave.

Acknowledgement. The authors would like to express their sincere thanks to Prof. H.-D. Alber and Prof. E. Zuazua for helpful discussions. This work has been partially supported by Grant MTM2008-03541 of the MICINN (Spain).

\section{References}

[1] Abeyaratne, R. and Knowles, J. (1990) On the driving traction acting on a surface of strain discontinuity in a continuum, J. Mech. Phys. Solids 38, 345-360.

[2] Alber, H.-D. (2000) Evolving microstructure and homogenization, Continuum. Mech. Thermodyn. 12, 235-287.

[3] Alber, H.-D. and Zhu, P. (2007) Evolution of phase boundaries by configurational forces, Archive Rat. Mech. Anal. 185, 235-286.

[4] Alber, H.-D., and Zhu, P. (2006) Solutions to a model with nonuniformly parabolic terms for phase evolution driven by configurational forces, SIAM J. Appl. Math. 66, No. 2, 680-699.

[5] Alber, H.-D., and Zhu, P. (2008) Solutions to a model for interface motion by interface diffusion, Proc. Roy. Soc. Edinburgh 138A, 923-955.

[6] Alber, H.-D., and Zhu, P. (2008a) Interface motion by interface diffusion driven by bulk energy: justification of a diffusive interface model, submitted to Conti. Mech. and Thermodyn..

[7] Allen, S. and Cahn, J. (1979) A microscopic theory for antiphase boundary motion and its application to antiphase domain coarsening, Acta Met. 27, 10841095. 
[8] Aronson, D. and Weiberger, H. (1978) Multidimensional nonlinear diffusions arising in population genetics, Adv. Math. 30, 33-76.

[9] Berestycki, H. and Hamel, F. (2007) Generalized travelling waves for reactiondiffusion equations, Perspectives in Nonlinear Partial Differential Equations. In honor of H. Brezis, Contemp. Mathematics, 446, Amer. Math. Soc., Providence, RI.

[10] Blesgen, T. and Weikard, U. (2005) Multi-component Allen-Cahn equation for elastically stressed solids, Electronic J. Diff. Eq.s, 2005, No. 89, 1-17.

[11] Cahn, J. (1959) Free Energy of a Nonuniform System. II. Thermodynamic Basis, J. Chem. Phys., 30, 1121-1124.

[12] Cahn, J. and Hilliard, J. (1959) Free Energy of a Nonuniform System. I. Interfacial Free Energy, J. Chem. Phys., 28, 258-267; Free Energy of a Nonuniform System. III. Nucleation in a Two-Component Incompressible Fluid, J. Chem. Phys., 31, 688-699.

[13] Cahn, J., and Taylor, J. (1994) Surface motion by surface diffusion. Acta Metall. Mater. 42, No. 4, 1045-1063.

[14] Fife, P. (1979) Mathematical aspects of reacting and diffusing systems, Springer Verlag.

[15] Fife, P. and McLeod, J. (1977) The approach of solutions of nonlinear diffusion equations to traveling front solutions, Arch. Rati. Mech. Anal., 65, 335-361.

[16] Gurtin, M. (2000) Configurational Forces as Basic Concepts of Continuum Physics, Springer Verlag, New York.

[17] Garcke, H. (2003) On the Cahn-Hilliard system with elasticity, Proc. Roy. Soc. Edinburgh, 133 A, 307-331.

[18] Hornbogen, E. and Warlimont, H. (2001) Metallkunde, 4th ed., Springer-Verlag.

[19] Kanel, Y. (1962) On the stabilization of solutions of the Cauchy problem for equations arising in the theory of combustion, Mat. Sbornik 59, 245-288.

[20] Mullins, W. (1957) Theory of thermal grooving. J. Appl. Phys. 28, No. 3, 333-339.

[21] Ninomiya, H. and Taniguchi, M. (2005) Existence and global stability of traveling curved fronts in the Allen-Cahn equations, J. Diff. Eq. 213, 204-233.

[22] Ninomiya, H. and Taniguchi, M. (2006) Global stability of traveling curved fronts in the Allen-Cahn equations, Disc. Conti. Dyna. Syst. 15, No. 3, 819-832.

[23] Pego, R. (1989) Front migration in the nonlinear Cahn-Hilliard equation, Proc. $R$. Soc. Lond. 422A, 261-278.

[24] Smoller, J. (1983) Shock waves and reaction-diffusion equations, Springer Verlag, New York

[25] Taniguchi, M. (2007) Traveling fronts of pyramidal shapes in the Allen-Cahn equations, SIAM J. Math. Anal. 39, No. 1, 319-344.

[26] Taylor, J. and Cahn, J. (1994) Linking anisotropic sharp and diffuse surface motion laws via gradient flows. J. Stat. Phys. 77, Nos. 1/2, 183-197.

[27] Yoshida, K. (1980) Functional analysis sixth edition, Springer Verlag, Berlin Heidelberg New York 

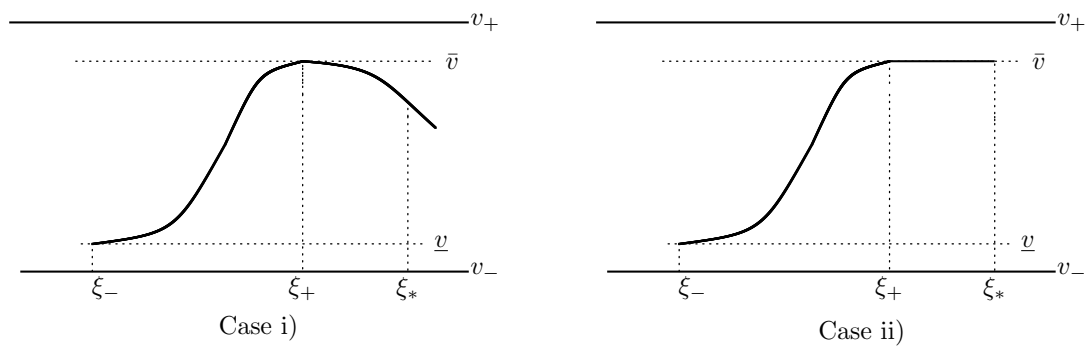

Figure 1. 